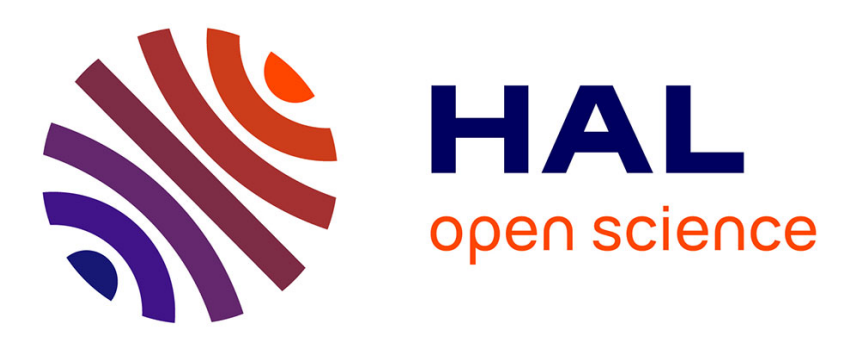

\title{
Multi-Objective Evolutionary Algorithms to Investigate Neurocomputational Issues: The Case Study of Basal Ganglia Models
}

\author{
Jean Liénard, Agnès Guillot, Benoît Girard
}

\section{To cite this version:}

Jean Liénard, Agnès Guillot, Benoît Girard. Multi-Objective Evolutionary Algorithms to Investigate Neurocomputational Issues: The Case Study of Basal Ganglia Models. From animals to animats 11: Simulation of Adaptive Behavior, Aug 2010, Paris, France. pp.597-606. hal-00645915

\section{HAL Id: hal-00645915 \\ https://hal.science/hal-00645915}

Submitted on 28 Nov 2011

HAL is a multi-disciplinary open access archive for the deposit and dissemination of scientific research documents, whether they are published or not. The documents may come from teaching and research institutions in France or abroad, or from public or private research centers.
L'archive ouverte pluridisciplinaire HAL, est destinée au dépôt et à la diffusion de documents scientifiques de niveau recherche, publiés ou non, émanant des établissements d'enseignement et de recherche français ou étrangers, des laboratoires publics ou privés. 


\title{
Multi-Objective Evolutionary Algorithms to Investigate Neurocomputational Issues : The Case Study of Basal Ganglia Models ${ }^{\star}$
}

\author{
Jean Liénard, Agnès Guillot and Benoît Girard \\ Institut des Systèmes Intelligents et de Robotique \\ Université Pierre et Marie Curie-Paris 6, CNRS UMR 7222 \\ 4, place Jussieu \\ 75252 Paris Cedex 05 - France \\ lienard@isir.upmc.fr
}

\begin{abstract}
The basal ganglia (BG) are a set of subcortical nuclei involved in action selection processes. We explore here the automatic parameterization of two models of the basal ganglia (the GPR and the CBG) using multi-objective evolutionary algorithms. We define two objective functions characterizing the supposed winner-takes-all functionality of the BG and obtain a set of solutions lying on the Pareto front for each model. We show that the CBG architecture leads to solutions dominating the GPR ones, this highlights the usefulness of the CBG additional connections with regards to the GPR. We then identify the most satisfying solutions on the fronts in terms of both functionality and plausibility. We finally define critical and indifferent parameters by analyzing their variations and values on the fronts, helping us to understand the dynamics governing the selection process in the BG models.
\end{abstract}

\section{Introduction}

In order to explore the characteristics and the critical parameter choices of models of the basal ganglia - a brain region involved in action selection- we use an empirical analysis method based on multi-objective evolutionary algorithms [1].

The Basal Ganglia (BG) is commonly defined as a set of subcortical interconnected nuclei, comprising the Striatum (Str), the Sub-Thalamic Nucleus (STN), both the external and internal Globus Pallidus (GPe and GPi) and both the Substantia Nigra par reticulata and pars compacta (SNr and SNc) [2]. The Str comprises mostly medium spiny neurons (MSN) and, more marginally, different types of interneurons, among which the fast-spiking interneurons (FSI) received special attention [3]. MSN are commonly subdivided on the basis of the presence of D1 or D2 dopamine receptors, distinguishing a striato-pallidal and a striatonigral pathway [4]. The BG form a loop with the ventro-lateral thalamus (VL), the thalamic reticular nucleus (TRN) and parts of the frontal cortex (FC).

* This research was funded by the ANR, project EvoNeuro ANR-09-EMER-005-01. 
The presented work is based on the hypothesis stipulating that the functional role of the BG is of generic action selection $[2,5]$. The BG are organized in multiple parallel segregated loops [6]. Within each of these loops, multiple striato-nigral channels interact, leading to a selection through disinhibition of the $\mathrm{GPi} / \mathrm{SNr}$, which is tonically active at rest [7]. This base level is strong enough to inhibit any action. The functionality of the BG would be to operate a "winnertakes-all" (WTA) algorithm, where the channel with the maximum input has the minimum output activity in the GPi/SNr (at least inferior to the base level), while the other ones have a maximal activity in the GPi/SNr (superior or equal to the base level). Based on a WTA hypothesis, many computational models have been proposed (see [8] for a recent review). These models are commonly set with hand-tuned parameters, with the goal of respecting biological constraints (for example, the STN has to be active at rest) while achieving a selection function, which is not necessarily expressed in precise quantitative terms.

Evolutionary Algorithms (EA) are designed to tackle optimization problems in a stochastic way. Inspired by the nature's evolutionary principle, the most striking difference to classical search is that EA evolve a population of solutions instead of a single one. Multi-Objective Evolutionary Algorithms (MOEA) are a subclass of EA conceived to address optimisation problems with multiple conflicting objectives [9]. Real world problems often involve a trade-off between antagonist goals, meaning they don't have a single best solution but admit a range of a priori equally acceptable solutions. Analyzing such a set of optimal solutions can help to better understand the dynamics governing the problem. Furthermore, one can eventually decide to pick one particular solution, based on expert knowledge.

There is no established method to parameterize a BG model. In a previous attempt at bringing mathematical techniques to elaborate neurocomputational models, Girard et al. [10] designed the BG with the help of the contraction theory to control its dynamics, de facto constraining the setting of parameters. Another recent attempt has been done by Wang et al. [11] by using a simple genetic algorithm and evolving the "GPR" model described in [12]. The main difference with our approach lies in their characterization of a WTA. They used a binary measure to reflect whether the inputs were classified correctly ( $i e$ whether the channel that should be selected is under an arbitrary threshold). We use here multiple scores to describe the comparative levels of disinhibition of all the channels; the goal here is to understand the parameterization compromises that are made when simultaneously trying to inhibit the loosing channels as much as possible, as well as disinhibiting the winning one.

\section{Materials \& Methods}

Building upon the recent "CBG" model [10] and the classical "GPR" model [12], we take their structures as a basis for two different evolutions. The connectivity of these models include the knowledge on the macro biology of the BG [13], the CBG including more of the known connectivity. Both of them incorporate the 
modeling of the thalamic loop, and both of them contain 6 concurrent striatonigral channels. In the GPR, each nucleus contains 6 classical leaky integrator neurons, whereas in the CBG each nucleus contains 6 locally Projected Dynamical System neurons, a leaky integrator variant with dynamics described in [10]. The evolution of the CBG and for the GPR can modify both the connections weights between nuclei (noted "nucleus $\rightarrow$ nucleus") and the tonic levels (noted " $\left.T_{\text {nucleus" }}\right)$. This accounts for a total of 25 parameters for the CBG, and 20 parameters for the GPR; see Figure 1 for the details of the connectivity, and Tables 2, 3 and 4 for the evolved parameters list.
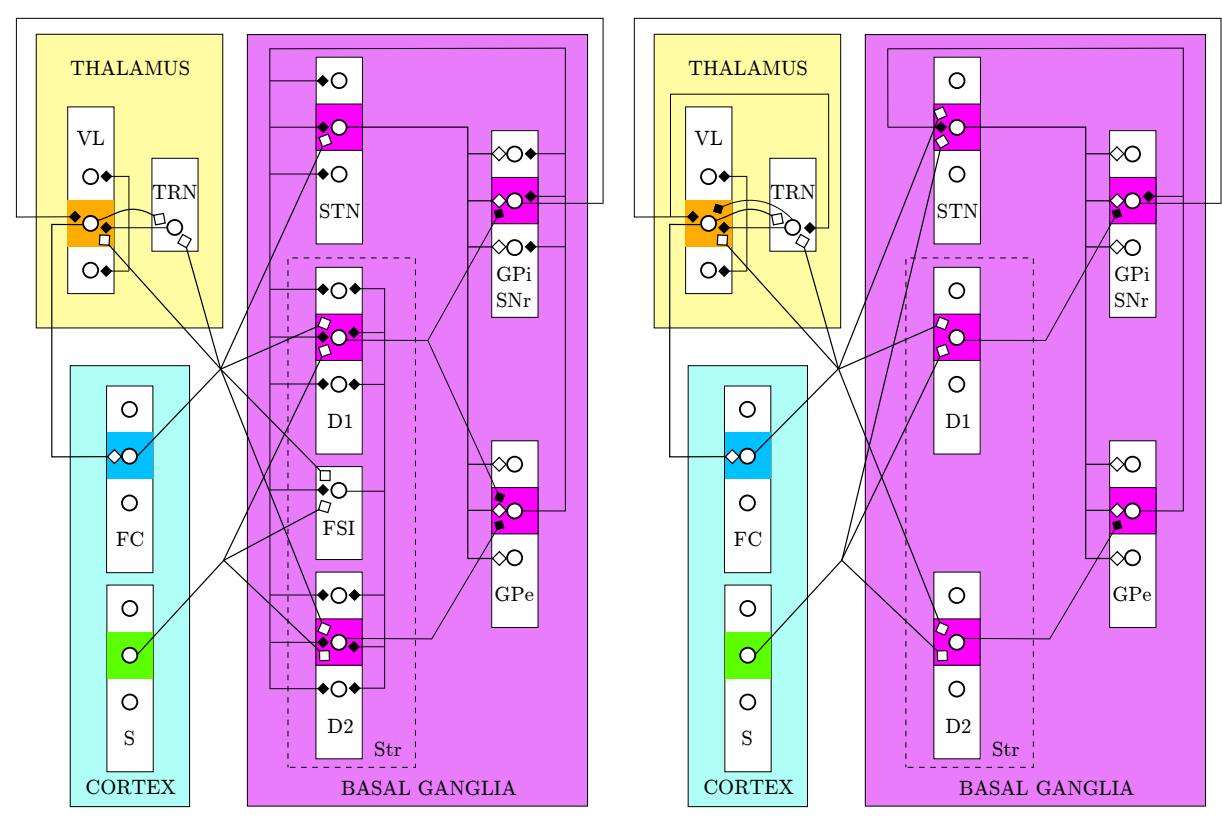

Fig. 1. The CBG (left) and GPR (right) architectures. Three channels in competition are represented. The outgoing connections of the shaded channel are the only one shown. White endings are excitatory, black are inhibitory. The inputs are the Saliences (S) in the cortex, and the outputs are the activity of the GPi/SNr neurons. See text for the meaning of other abbreviations.

We introduced some constraints on the parameters, in order to be sure that some levels of biological plausibility are respected but also to reduce the exploration space to make the convergence of the evolution more likely. Neurons from the GPe and GPi exhibit comparable baseline [14], hence they have the same tonic levels : $T_{\mathrm{GPe}}=T_{\mathrm{GPi}}$. Neurons of the Striatum with D1 and D2 receptors are thought to have comparable afferents from the Cortex [15], so $S \rightarrow D 1=S \rightarrow D 2$ and $F C \rightarrow D 1=F C \rightarrow D 2$. Afferents to both 
the GPe and GPi from the STN appear to be the same [16], consequently $S T N \rightarrow G P e=S T N \rightarrow G P i$. To be acceptable, a solution had to have a GPi output at rest larger than 0.09, as the GPi is known to be tonically active [2] and as this output is 0.10 for the CBG and 0.17 for the GPR. Finally, we chose to limit connection weights in the range $[0.05 ; 1]$. Indeed, a null connection weight would mean no connection at all, changing the connectivity of the circuit.

\subsection{Evolving the circuit}

Our choice for simulating the evolution is the widely used NSGA-II algorithm [17]. The mutation rate was fixed at $\mu=0.1$, a rather high value that serves to widely explore the space of parameters. We set the distribution index for mutation and cross-over operators in favor of mutation, with $\eta_{m}=15$ and $\eta_{c}=$ 10. We scheduled 10 runs comprising each 1500 generations, with a population size of 200. The computational framework used was SFERES [18]. Designed to work in a parallel fashion, it permits fast simulation on multi-cores machines; it is also fairly easy to set up and use.

The selection functionality of the BG has to be formally defined so as to allow the computation of some fitness criteria. To evaluate the quality of the circuits, they were submitted to $N=500$ different random inputs drawn from an uniform distribution. They were simulated during one second with each of these inputs, before the outputs are considered for fitness evaluation.

Two objectives have been defined for the evolution. First, the channel corresponding to the largest input (the selected channel) has to be disinhibited to the maximum. This translates straightforwardly as :

$$
\text { First objective : minimise } f_{1}=\frac{\sum_{N} G P I_{\text {selected channel }}}{N}
$$

But this is not sufficient to obtain a WTA algorithm, as this could lead to the disinhibition of all channels. Therefore, we define the second objective as the mean of the five other channels :

Second objective : maximise $f_{2}=\frac{\sum_{N}\left(\sum_{\text {channel } \neq \text { selected channel }} \frac{G P i_{\text {channel }}}{5}\right)}{N}$

\section{Results}

Each run resulted after 1500 generations in a set of non-dominated solutions, called a Pareto front [9]. We define the global Pareto-optimal front as the set of non-dominated solutions from all the runs (Figure 2). Every front contributes to it for both models (Table 1), hence we assume they all have converged to the same optimal front for both experiments. 


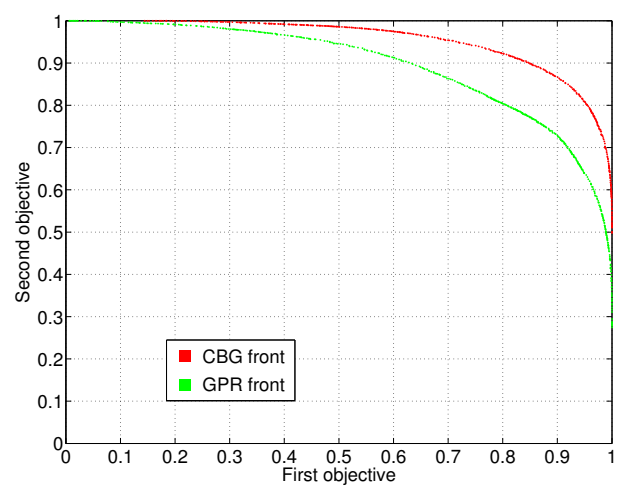

Fig. 2. The fitnesses for the Pareto-fronts of the evolution of CBG and GPR.

Table 1. Percentages of contribution to the pareto-optimal front for each run

\begin{tabular}{lllllllllll} 
run & 1 & 2 & 3 & 4 & 5 & 6 & 7 & 8 & 9 & 10 \\
\hline CBG & $11 \%$ & $9 \%$ & $18 \%$ & $8 \%$ & $10 \%$ & $16 \%$ & $6 \%$ & $4 \%$ & $13 \%$ & $4 \%$ \\
GPR & $14 \%$ & $8 \%$ & $17 \%$ & $5 \%$ & $14 \%$ & $8 \%$ & $7 \%$ & $3 \%$ & $8 \%$ & $17 \%$
\end{tabular}

As the best solutions are within the global Pareto-optimal fronts, we will confine further development to them. Each of these solutions represents the best trade-off from a certain point of view; a priori there is no reason to chose one particular solution at this level of analysis. The Pareto-optimal front comprises 510 solutions for the CBG and 551 solutions for the GPR. Interestingly, solutions from the CBG front dominate those from the GPR front (Figure 2), the additional degrees of freedom of the CBG can thus be exploited to globally improve the performance on our task.

Overview of the parameters. For the CBG, 8 parameters are maximised or minimised for all the solutions (Table 2); for the GPR, only two parameters are in this case (Table 3). The dispersion of a parameter for close solutions on the front indicates how much this parameter contributes to the fitnesses (high dispersion reflect minor importance). The standard deviation (SD) does not suffice to express this, as it is a global indicator reflecting the dispersion of the values across the whole front. Hence we use the standard deviation of the first difference of the parameter values accross the front.

For both models, no parameter is at the boundaries in the thalamic loop, and the SD is high as it ranges from 0.23 to 0.29 (Table 4). The first difference $\mathrm{SD}$ is high too, ranging from 0.33 to 0.42 . This shows high dispersion for close solutions. Hence the contribution of each of these parameters to the fitness scores appears as secondary, as the evolution process did not set them to precise values.

WTA functionality analysis. We designed the two objectives so that their combination favors the WTA functionality. Of course, maximizing only one of 
Table 2. Mean $\pm \mathrm{SD}$ and first difference SD for the CBG parameters (except the thalamic loop)

\begin{tabular}{lcc}
\multicolumn{3}{c}{ Minimised } \\
\hline$F C \rightarrow F S$ & $0.05 \pm 0.01$ & 0.01 \\
$G P e \rightarrow D 2$ & $0.06 \pm 0.05$ & 0.04 \\
$G P e \rightarrow G P i$ & $0.07 \pm 0.03$ & 0.01 \\
$-T_{D 1 / D 2}$ & $0.06 \pm 0.02$ & 0.03 \\
\hline \multicolumn{3}{c}{ Maximised } \\
\hline$S \rightarrow D 1 / D 2$ & $1 \pm 0.01$ & 0.01 \\
$D 1 \rightarrow G P i$ & $1 \pm 0.01$ & 0.02 \\
$D 2 \rightarrow G P e$ & $0.99 \pm 0.04$ & 0.05 \\
$T_{G P e / G P i}$ & $0.98 \pm 0.03$ & 0.04 \\
\hline
\end{tabular}

\begin{tabular}{lcc}
\multicolumn{3}{c}{ Between the limits } \\
\hline$S \rightarrow F S$ & $0.74 \pm 0.14$ & 0.17 \\
$G P e \rightarrow S T N$ & $0.15 \pm 0.04$ & 0.03 \\
$G P e \rightarrow D 1$ & $0.47 \pm 0.11$ & 0.05 \\
$G P e \rightarrow F S$ & $0.34 \pm 0.07$ & 0.09 \\
$S T N \rightarrow G P e / G P i$ & $0.45 \pm 0.35$ & 0.14 \\
$D 1 \rightarrow G P e$ & $0.76 \pm 0.22$ & 0.12 \\
$F S \rightarrow D 1 / D 2$ & $0.48 \pm 0.22$ & 0.09 \\
$F C \rightarrow D 1 / D 2$ & $0.43 \pm 0.25$ & 0.11 \\
$F C \rightarrow S T N$ & $0.24 \pm 0.17$ & 0.16 \\
$T_{S T N}$ & $0.91 \pm 0.13$ & 0.17 \\
\hline
\end{tabular}

Table 3. Mean \pm SD and first difference SD for the GPR parameters (except the thalamic loop)

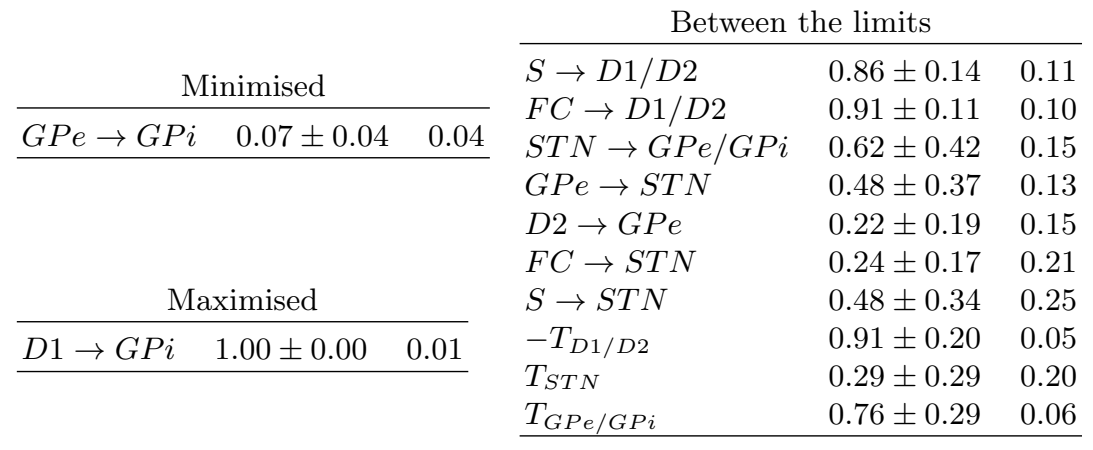

these objectives is useless for that: a circuit systematically disinhibiting all the channels would have a score $\left(f_{1}=1, f_{2}=0\right)$, while a circuit maximally inhibiting all the channels would have a score $\left(f_{1}=0, f_{2}=1\right)$. As shown in Figure 2 , the evolution found no solution that can maximise both of these objectives.

In order to more deeply characterize the solutions, we studied their relationship with the base level, defined as the output of the GPi/SNr with null inputs. Electrophysiological studies show that the GPi is tonically active in the absence of inputs, meaning that the base level should be high [2]. Furthermore, when there are inputs to the BG, most of the GPi neurons have an increasing activity compared to the resting state, and a few have a decreasing activity [19]. Hence, as mentioned in introduction, a biologically plausible selection should have a base level higher than the selected channel's output (expressed by objective 1) and lower than the mean of unselected channels output (expressed by objective 2). Figure 3 (top) represents the objectives functions altogether with the base level for each solution of the fronts. The values of the base levels seem to be structured with regard to fitness values, plateaus of specific values appear, especially a very constrained one for the CBG individuals in the 250-450 interval. With regards 
Table 4. Mean \pm SD and first difference SD for the thalamic loop parameters

\begin{tabular}{lcc}
\multicolumn{3}{c}{ CBG parameters } \\
\hline$B G \rightarrow V L$ & $0.32 \pm 0.24$ & 0.33 \\
$F C \rightarrow V L$ & $0.54 \pm 0.28$ & 0.38 \\
$F C \rightarrow T R N$ & $0.52 \pm 0.29$ & 0.4 \\
$V L \rightarrow F C$ & $0.47 \pm 0.26$ & 0.35 \\
$V L \rightarrow T R N$ & $0.62 \pm 0.27$ & 0.38 \\
$T R N \rightarrow V L$ & $0.63 \pm 0.29$ & 0.42 \\
$T_{V L}$ & $0.44 \pm 0.24$ & 0.34 \\
\hline
\end{tabular}

\begin{tabular}{lcc}
\multicolumn{3}{c}{ GPR parameters } \\
\hline$B G \rightarrow V L$ & $0.53 \pm 0.27$ & 0.37 \\
$B G \rightarrow T R N$ & $0.53 \pm 0.24$ & 0.34 \\
$F C \rightarrow V L$ & $0.57 \pm 0.27$ & 0.38 \\
$F C \rightarrow T R N$ & $0.44 \pm 0.24$ & 0.33 \\
$V L \rightarrow F C$ & $0.62 \pm 0.26$ & 0.34 \\
$V L \rightarrow T R N$ & $0.53 \pm 0.25$ & 0.34 \\
$T R N \rightarrow V L$ & $0.56 \pm 0.27$ & 0.39 \\
$T R N \rightarrow V L_{\text {self }}$ & $0.52 \pm 0.23$ & 0.33 \\
\hline
\end{tabular}

to the aforementioned biological plausibility criterion, the best solutions for the CBG are those ranging approximately from 1 to 100 and from 250 to 450 . For the GPR, they are the ones from 1 to 150 and those larger than 325 .

We can further refine the set of acceptable solutions by imposing an exclusive selection. Indeed, we did not explicitely forbid the designation of multiple winners; some of the solutions obtained often disinhibit more than one channel, instead of the one with the most important input only. The percentage of such multiple selections for each solution is represented in Figure 3 (bottom). Interestingly, the best selection is found for the solutions operating a biologically plausible WTA (solutions 250 to 450 for the CBG and 325 to 450 for the GPR), comforting us in the adequacy between the supposed function operated by the BG, the modeling of the BG (as described by the CBG or GPR) and electrophysiological data.

\section{Discussion}

In this work, we show that two existing models of BG $[10,12]$ can be optimized through MOEA, with the goal of recreating a "winner-takes-all" behavior. The analysis of the variations of the parameters of the best solutions highlighted some regularities and helped identify critical and indifferent parameters. The study of the biological plausibility of the solutions helped identifying the most interesting parts of the Pareto front. Generality of the reasoning is exemplified by the application on two different models.

Parameterization exploration. On the basis of this case study, it seems that the MOEA permit to gain insight into the properties of models of a given neural structure. By generating multiple solutions fulfilling antagonist objectives, MOEA can be used as a mean for exploration. In this paper, we divided the modeling of a WTA into two separate sub-problems (expressed by equations 1 and 2) to explore a wide variety of different WTA implementations. It is then possible to pick particular solutions on the basis of "expert knowledge" not 

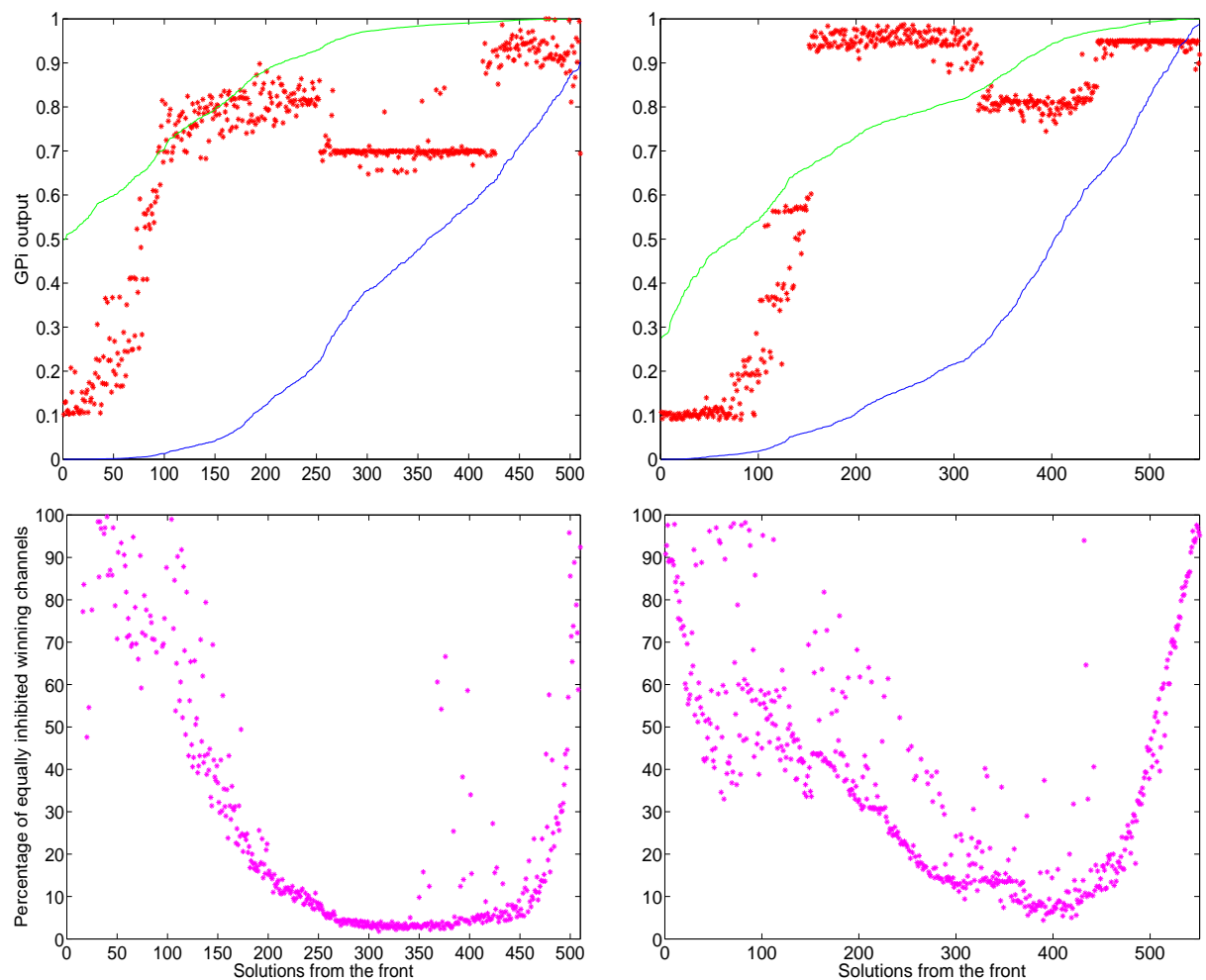

Fig. 3. Left graphics concern the CBG, right graphics concern the GPR. The horizontal abscisse of each graphic corresponds to the solutions along the front. Top : blue and green lines are the values for $f_{1}$ and $f_{2}$ respectively (equations 1 and 2 ); red dots represent the base level. Bottom : points represent the percentage of cases where two channels are equally disinhibited, leading to an abusive selection of both of them.

expressed in the constraints, as we did by delineating a biologically plausible selection behavior.

Model comparison. Comparison between two architectures is also possible. The CBG Pareto front dominates the GPR one (Figure 2). Furthermore, the percentage of cases with two winning channels instead of one (bottom of Figure 3 ) is smaller for the CBG. Hence, the additional degrees of freedom permitted by the CBG (Figure 1) are useful for our purposes, as they lead to a better adequacy to a WTA function.

Parameter contribution. The MOEA help identifying the contribution of a given parameter to a particular behavior. Maximised parameters along the front give informations on the most important parameters needed to achieve a 
presupposed function. For the CBG, these parameters are $S \rightarrow D 1 / D 2, D 1 \rightarrow$ $G P i, D 2 \rightarrow G P e$ and $T_{G P e / G P i}$ (Table 2). This outlines some of the principal connections of the Direct / Indirect pathway hypothesis [20], comforting in some sense the fundamental pertinence of these two loops in order to achieve selection. Interestingly, the less complete GPR structure does not permit to see this, as the $D 2 \rightarrow G P e$ connection is limited (Table 3 ).

Minimised parameters indicates that they are in opposition with the desired behavior. In both models, the $G P e \rightarrow G P i$ connection is minimised. On one hand, this is contradictory with anatomical data pointing out that one third of the projecting GPe neurons target the GPi/SNr [21], implying that the connection from GPe to GPi is not non-existent. On the other hand, electrophysiological data lead to the hypothesis that they might be weak [22]. Our contribution to this precise point is that, in all the variations of WTA obtained with both the CBG and GPR structures, this connection grieves the supposed functionality. To explain this result, we can conceive three non-contradictory hypothesis : (1) both the CBG and GPR lack in the modeling of a (perhaps unknown) fundamental connection or property enabling the $G P e \rightarrow G P i$ to contribute to a WTA algorithm; (2) the BG is performing another function which gives meaning to this connection; (3) in accordance with electrophysiological data, this connection is effectively weak.

The study of the parameter variances for solutions exhibiting comparable fitnesses permits to isolate parameters that are not needed for the desired behavior. The thalamic loop of the CBG and GPR models has a high first difference SD (Table 4), hence the modeling of these is not pertinent with regards to the defined objectives. This is coherent with the intuition of the modelers [23] who used it to modulate the time taken for the selection. Defining a third objective for the time of convergence could help to explore the thalamic loop role.

Future work will first involve examining in details what in the BG model structure underlies the appearance of a base level plateau for the best WTA solutions obtained, and its possible relevance for the functional neurobiology of the BG. We will also dig deeper into the biological plausibility of BG models. By adding connections and neuronal properties neglected in BG models and by polishing our set of constraints, we will evolve a more complete model with the goal of imitating data from electrophysiological experiments and make new predictions on the role of various BG connections.

\section{References}

1. Doncieux, S.: Evolutionary algorithms as exploration and analysis helper tools, application to a flapping wings aircraft. In: IROS Workshop "Exploring New Horizons in Evolutionary Design of Robots", Saint Louis, USA (2009) 19-25

2. Mink, J.: The basal ganglia: focused selection and inhibition of competing motor programs. Progress in Neurobiology 50(4) (1996) 381-425

3. Tepper, J., Bolam, J.: Functional diversity and specificity of neostriatal interneurons. Current opinion in neurobiology 14(6) (2004) 685-692 
4. Gerfen, C., Engber, T., Mahan, L., Susel, Z., Chase, T., Monsma Jr, F., Sibley, D.: D1 and D2 dopamine receptor-regulated gene expression of striatonigral and striatopallidal neurons. Science 250(4986) (1990) 1429

5. Redgrave, P., Prescott, T., Gurney, K.: The basal ganglia: a vertebrate solution to the selection problem? Neuroscience-Oxford 89 (1999) 1009-1024

6. Alexander, G., DeLong, M., Strick, P.: Parallel organization of functionally segregated circuits linking basal ganglia and cortex. Annual review of neuroscience 9(1) (1986) 357-381

7. Chevalier, G., Deniau, J.: Disinhibition as a basic process in the expression of striatal functions. Trends Neurosci 13(7) (1990) 277-280

8. Cohen, M., Frank, M.: Neurocomputational models of basal ganglia function in learning, memory and choice. Behavioural brain research 199(1) (2009) 141-156

9. Deb, K.: Multi-objective optimization using evolutionary algorithms. Wiley (2001)

10. Girard, B., Tabareau, N., Pham, Q., Berthoz, A., Slotine, J.: Where neuroscience and dynamic system theory meet autonomous robotics: a contracting basal ganglia model for action selection. Neural Networks 21(4) (2008) 628-641

11. Wang, Y., Li, S., Chen, Q., Hu, W.: Biology Inspired Robot Behavior Selection Mechanism: Using Genetic Algorithm. Lecture notes in computer science 4688 (2007) 777

12. Gurney, K., Prescott, T., Redgrave, P.: A computational model of action selection in the basal ganglia. I. A new functional anatomy. Biological cybernetics 84(6) (2001) 401-410

13. Tepper, J., Abercrombie, E., Bolam, J.: Basal ganglia macrocircuits. Progress in brain research 160 (2007) 3

14. Turner, R., Anderson, M.: Pallidal discharge related to the kinematics of reaching movements in two dimensions. Journal of neurophysiology 77(3) (1997) 1051

15. Ballion, B., Mallet, N., Bezard, E., Lanciego, J., Gonon, F.: Intratelencephalic corticostriatal neurons equally excite striatonigral and striatopallidal neurons and their discharge activity is selectively reduced in experimental parkinsonism. European Journal of Neuroscience 27(9) (2008) 2313-2321

16. Shink, E., Bevan, M., Bolam, J., Smith, Y.: The subthalamic nucleus and the external pallidum: two tightly interconnected structures that control the output of the basal ganglia in the monkey. Neuroscience 73(2) (1996) 335-357

17. Deb, K., Pratap, A., Agarwal, S., Meyarivan, T., Fast, A., Algorithm, E.: NSGA-II. IEEE transactions on evolutionary computation 6(2) (2002)

18. Mouret, J.B., Doncieux, S.: Sferes ${ }_{\mathrm{v} 2}$ : Evolvin' in the multi-core world (submitted). (2010)

19. Gulley, J., Kuwajima, M., Mayhill, E., Rebec, G.: Behavior-related changes in the activity of substantia nigra pars reticulata neurons in freely moving rats. Brain research 845(1) (1999) 68-76

20. Albin, R., Young, A., Penney, J.: The functional anatomy of basal ganglia disorders. Trends in Neurosciences 12(10) (1989) 366-375

21. Sato, F., Lavallee, P., Levesque, M., Parent, A.: Single-axon tracing study of neurons of the external segment of the globus pallidus in primate. Journal of Comparative Neurology 417(1) (2000) 17-31

22. Nambu, A.: Seven problems on the basal ganglia. Current Opinion in Neurobiology 18(6) (2008) 595-604

23. Girard, B., Cuzin, V., Guillot, A., Gurney, K.N., Prescott, T.J.: A basal ganglia inspired model of action selection evaluated in a robotic survival task. Journal of Integrative Neuroscience 2(2) (2003) 179-200 